\title{
Pediatric hydrocephalus: systematic literature review and evidence-based guidelines. Part 1: Introduction and methodology
}

\author{
Ann Marie Flannery, M.D., ${ }^{1}$ And Laura Mitchell, M.A. ${ }^{2}$ \\ ${ }^{1}$ Department of Neurological Surgery, Saint Louis University, St. Louis, Missouri, and ${ }^{2}$ Guidelines \\ Department, Congress of Neurological Surgeons; Schaumburg, Illinois
}

\begin{abstract}
This clinical systematic review of and evidence-based guidelines for the treatment of pediatric hydrocephalus were developed by a physician volunteer task force. They are provided as an educational tool based on an assessment of current scientific and clinical information as well as accepted approaches to treatment. They are not intended to be a fixed protocol, because some patients may require more or less treatment.

In Part 1, the authors introduce the reader to the complex topic of hydrocephalus and the lack of consensus on its appropriate treatment. The authors describe the development of the Pediatric Hydrocephalus Systematic Review and Evidence-Based Guidelines Task Force charged with reviewing the literature and recommending treatments for hydrocephalus, and they set out the basic methodology used throughout the specific topics covered in later chapters. (http://thejns.org/doi/abs/10.3171/2014.7.PEDS14321)
\end{abstract}

KEY WORDS - hydrocephalus systematic evidence review
- $\quad$ practice guidelines
$\mathrm{P}$ EDIATRIC hydrocephalus is the most common surgically correctable neurological problem in infants, children, and adolescents. It is estimated that hydrocephalus may occur with the frequency of 1 in every 500 children. There are multiple causes of this disorder: genetic causes such as X-linked aqueductal stenosis; other congenital causes including myelomeningocoele and Chiari malformation; and acquired causes such as intraventricular hemorrhage, trauma, tumors, and infection. The burden of disease is substantial: hydrocephalus can have an effect on development as well as an impact on overall quality of life. Children with hydrocephalus represent a disproportionate share of all children admitted to hospitals. ${ }^{5}$ There are effective surgical interventions that can preserve and improve quality of life; however, these are not without side effects and failures. The need for improvements in surgical interventions is well understood.

Patients, families, and treating physicians acknowledge that, while the problems of hydrocephalus appear

\footnotetext{
Abbreviations used in this paper: AANS = American Association of Neurological Surgeons; CNS = Congress of Neurological Surgeons; JGC = Joint Guidelines Committee.
}

simple, they are actually quite complex. Current treatment methods are insufficient, and there is currently little agreement on the "best" treatment, even among leading practitioners. Nevertheless, there is consensus that current management may result in "frequent complications, poor rate of shunt survival, and poor quality of life for patients leading to unsatisfactory outcomes."

Recent publications on readmission rates associated with pediatric hospitalization have only served to highlight the need for close scrutiny of current interventions. Ventriculoperitoneal shunt procedures account for only a small number of the total admissions analyzed, but they have the second highest readmission prevalence. As the focus on prevention of hospital readmission increases, this information further emphasizes the importance of discerning safe and effective treatments for hydrocephalus. ${ }^{1}$

This review was conducted to evaluate the best available evidence to aid clinicians and to guide clinical practice by determining the best options for the management of pediatric hydrocephalus. The details of specific topics selected will be discussed in the following papers, but, in general, in their analyses members of the Pediatric Hydrocephalus Systematic Review and Evidence-Based 
Guidelines Task Force looked at available treatments, the effects and complications of those treatments, and strategies to avoid and manage complications. In undertaking this review, we define the condition "hydrocephalus" for purposes of the literature search and use the term "pediatric" to specify the care of infants, children, and adolescents younger than 18 years of age. The scope of this inquiry will include patients with either congenital or acquired hydrocephalus, including communicating and noncommunicating hydrocephalus for which the causes are undetermined. This review was undertaken under the auspices of the Pediatric Section of the American Association of Neurological Surgeons (AANS) and the Congress of Neurological Surgeons (CNS).

The recommendations contained in this supplement to the Journal of Neurosurgery: Pediatrics deliberately eschew the use of expert opinion, relying strictly on information available in the literature. Studies have reported that expert opinions may not use evaluable evidence, if the papers containing that evidence do not support the "expert" point of view. ${ }^{2}$ Throughout the development of these guidelines, the Task Force used evidence-based methodologies and adhered to strict criteria that had been defined a priori as specified by the Institute of Medicine's standards for conducting systematic reviews and clinical evidence-based guidelines, as well as the methodologies described below.

This effort was begun by a small study group that convened at the Pediatric Section Annual Meeting in Austin, Texas, in 2011. At that time the basic topics were considered, and over the course of several months these were further refined. The search strategies we used will be discussed in detail in a later section. Members of the Task Force involved in the creation of this document were recruited from a variety of institutions and subspecialty disciplines in an effort to have as broad a representation of opinions and expertise as possible. Pediatric neurosurgeons, their patients, and patients' family members hope that someday hydrocephalus will be entirely preventable or curable. Until that time, efforts must continue to refine and improve treatment as well as the evaluation of the effectiveness of treatment.

The Task Force followed protocols established by the Joint Guidelines Committee (JGC) of the AANS and the CNS. A conscientious effort was also made to be sure that conflict of interest was avoided. Members who had published extensively in certain areas were mindfully assigned to evaluate evidence in other topics. Every effort was made to ensure that the work product would be transparent and trustworthy. ${ }^{4}$

\section{Methods}

\section{Process Overview}

The Task Force and the Pediatric Section of the AANS/CNS conducted a systematic review of the literature relevant to the management of hydrocephalus in infants and children. Additional details of the systematic review are provided below. During the development process, the panel participated in a series of conference calls and meetings. Multiple iterations of the written review were conducted by individuals in the Task Force and various AANS/CNS committees (Fig. 1).

\section{Selection of Clinical Topics}

The goals of this effort were to discern the most effective strategies for a variety of hydrocephalus-related problems, including acquired hydrocephalus of the premature neonate. We also considered the use of technical adjuvants such as antibiotic-impregnated catheters, endoscopic placement of shunt catheters, electromagnetic guidance for shunt catheter placement, and ultrasound guidance for shunt catheter placement. It was hoped that these adjuvants would lead to improvements in outcome and a reduction in the frequency of revision.

Complications associated with ventriculoperitoneal shunts and endoscopic third ventriculostomies are known, and these interventions' effects and long-term successes are useful to evaluate. Complications associated with infection are of particular significance. Therefore, the prevention and treatment of infection occupies a significant portion of the hydrocephalus literature. Finally, the correlation of ventricle size to outcome in a child is a source of great interest as an indicator of the success of the intervention.

Following the identification of hydrocephalus-related problems, the Task Force developed preliminary recommendations that were formatted similarly to the PICO (patients, interventions, comparisons, and outcomes) formula to aid in the determination of the overall scope of the review and the terminology used to formulate the literature search strategies described below.

\section{Literature Search}

The Task Force worked with a research librarian and methodologist to assist with the formulation of search terms and strategies used to search the US National Library of Medicine PubMed/MEDLINE database and the Cochrane Database of Systematic Reviews for relevant literature published between January 1966 and March 2012.

Four to five Task Force members used the article inclusion/exclusion criteria described below to screen abstracts and provide a list of relevant articles for a full-text review. Each Task Force member was blinded to the lists of abstracts provided by others. Staff compiled all lists together for review and final approval by all Task Force members.

The searches were supplemented with manual screenings of bibliographies from all retrieved articles. In addition, the bibliographies of potentially relevant systematic reviews were screened for potentially relevant articles. All literature identified either by searches of the electronic database or by manual searches were subject to the article inclusion/exclusion criteria listed below. Specific search strategies used by Task Force members are provided within the Methods sections of the topics evaluated later in this supplement.

\section{Article Inclusion/Exclusion Criteria}

Articles were retrieved and included as evidence to support the topics discussed in this review if they met specific inclusion/exclusion criteria. These criteria were 

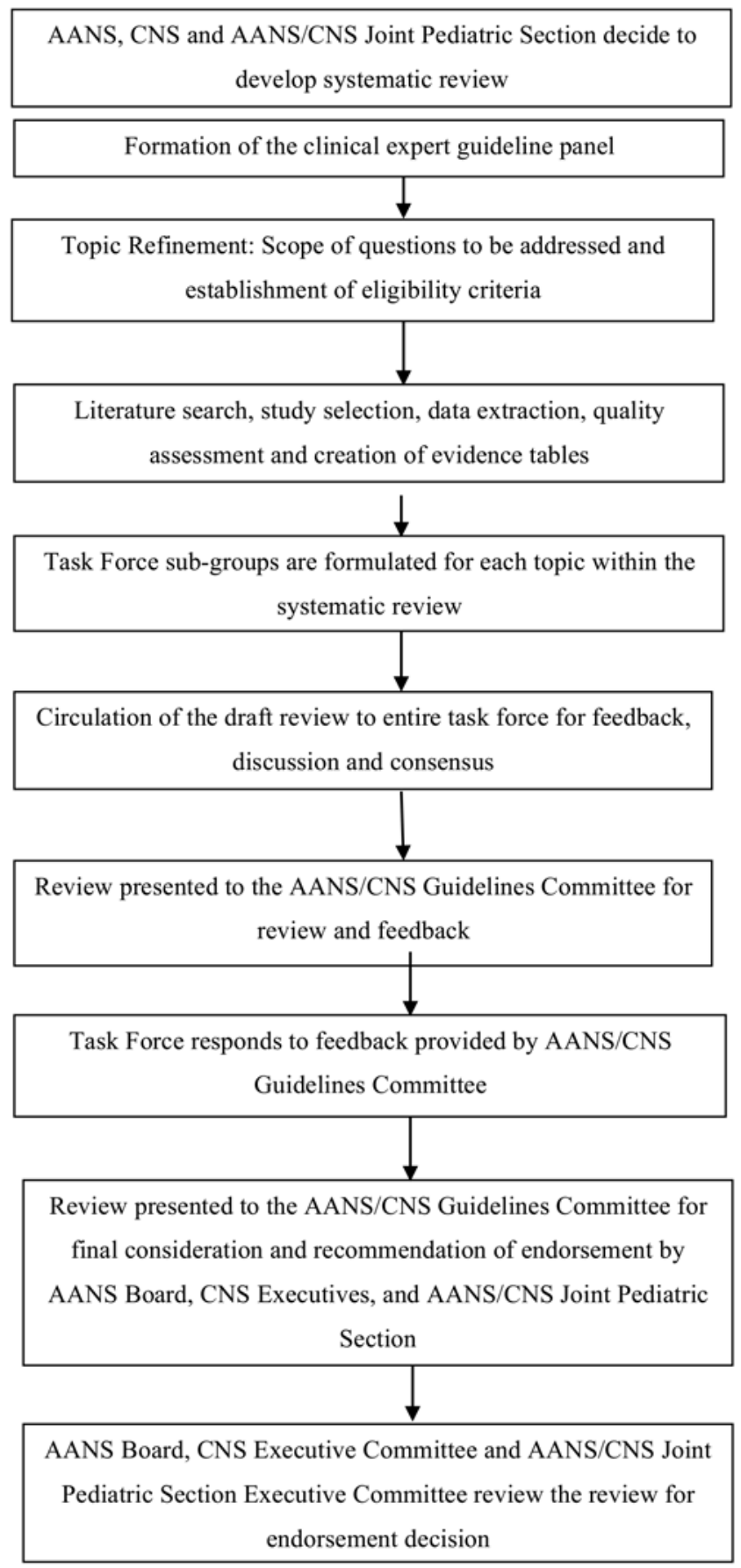

FIG. 1. Flowchart showing the overall development process.

also applied to articles provided by Task Force members who supplemented the electronic database searches with articles obtained from manual searches of bibliographies from the original articles. To reduce bias, the criteria were specified before conducting the literature searches. For the purposes of the systematic review and guidelines, articles that did not meet the following criteria were not deemed evidence and were not considered as potential evidence to support the topics and clinical recommendations.
To be included in our review, an article had to meet the following criteria:

- Studies that combined results in patients (younger than 18 years of age) who had congenital and acquired hydrocephalus with results in patients with "normal" pressure hydrocephalus were excluded if the study enrolled fewer than $80 \%$ of the target patient population.

- Studies that enrolled mixed patient populations were included only if separate results were reported for the target population. The results of the target population were the only results considered as evidence to support our recommendations.

- The study was a full article report of a clinical study.

- The study was not a meeting abstract, editorial, letter, or a commentary.

- Prospective case series had to report baseline values.

- Case series studies with nonconsecutive enrollment of patients were excluded. (This was determined by a review of the Methods section in the relevant article.)

- Studies had to have appeared in a peer-reviewed publication or a registry report.

- Studies had to enroll at least 10 patients for each distinct outcome that was measured. If a comparative study, a minimum enrollment of five patients per treatment arm for each outcome was necessary.

- The study involved humans.

- The study was published in or after 1966.

- The study presented results quantitatively.

- The study did not involve "in vitro" or "biomechanical" data or results obtained in cadavers.

- The study was published in English.

- Papers reporting the results of systematic reviews, meta-analyses, or guidelines developed by others were excluded.

Articles presenting systematic reviews or meta-analyses conducted by others, as well as guidelines developed by others, were not included as evidence to support this review due to differences in inclusion/exclusion criteria between those specified in such articles and those established by the Task Force. Although such articles were not included as evidence to support the review, they were recalled for full-text review so that the Task Force could conduct manual searches of the articles' bibliographies.

\section{Statistical Methods}

For some topics, the available literature provided sufficient quality and quantity of data to allow more detailed statistical analysis going beyond the basic methods described in this paper. For those topics, including the ones shown in Part $6^{4}$ and Part $7,{ }^{3}$ the methods that were used will be described separately. In brief, a forest plot was created and a meta-analysis was conducted to determine the overall effect of the intervention, such as preoperative antibiotics, after the sources had been selected using evidencebased criteria, as described above.

\section{Revision Plans}

In accordance with the Institute of Medicine's standards for developing clinical practice guidelines and cri- 
teria specified by the National Guideline Clearinghouse, following the release of this document the Task Force will monitor related publications and will revise the entire document and/or specific sections "if new evidence shows that a recommended intervention causes previously unknown substantial harm; that a new intervention is significantly superior to a previously recommended intervention from an efficacy or harms perspective; or that a recommendation can be applied to new populations." In addition, the Task Force will confirm within 5 years following the date of publication that the content is up to date with current clinical practice and available technologies for the treatment of pediatric hydrocephalus.

\section{Rating the Quality of Evidence}

The quality of evidence was rated using an evidence hierarchy developed by the AANS/CNS Guidelines Committee for each of the four different study types: therapeutic, diagnostic, prognostic, and clinical assessment. Additional information regarding the hierarchy classification of evidence can be located here: http://www.cns.org/advocacy/ jgc/pdf/JGCGuidelineDevelopmentMethodology.pdf.

\section{Strength of the Recommendations Rating Scheme}

The Task Force used methodologies endorsed by the AANS/CNS Guidelines Committee to assign a strength category to each recommendation in this review. Linking evidence to recommendations through the use of evidentiary tables has been endorsed by the American Medical Association, the AANS, and the CNS. This process validates and supports the relationship between the strength of evidence and the strength of recommendations. Demonstrating the highest degree of clinical certainty, Class I evidence is used to support recommendations of the strongest type, defined as Level I recommendations. Level II recommendations reflect a moderate degree of clinical certainty and are supported by Class II evidence or a strong consensus of Class III evidence. Level III recommendations denote clinical uncertainty, which is supported by inconclusive or conflicting evidence or expert opinion.

\section{Voting on the Recommendations}

The Task Force used a structured voting technique to finalize and approve the final recommendations, language, and strength of the recommendations presented in this review. The voting technique is referred to as the "nominal group technique" and is described in an article by Murphy et al. ${ }^{5}$ This technique includes up to 3 rounds of voting using secret ballots to ensure that each Task Force member is blinded to the responses of other Task Force members. All the recommendations in this review were approved following the first round of voting and no further discussion was needed to finalize the following recommendations. During the course of editing and finalizing the document, changes were made to allow recommendations to conform to the rules of evidence and language as described earlier. When this occurred, the changes were reviewed and approved by the group.

\section{Guideline Panel Consensus and Practice Guideline Approval Process}

Topic subtask forces were created from the larger Task Force. Each subtask force took part in the literature selection, review of the literature, creation of the evidence tables, and creation and editing of the final review. The final draft review was then circulated to the entire Task Force for feedback, discussion, and, ultimately, approval.

Following Task Force approval, the completed systematic review was presented to the JGC of the AANS and CNS for consideration and recommendation of endorsement on behalf of the CNS Executive Committee and the AANS Board of Directors. As part of the evaluation process, the JGC reviewers could provide input on the content and methodologies used to create the systematic review.

Development of this review was editorially independent from the funding agencies (the CNS Executive Committee and the AANS/CNS Joint Pediatric Section Executive Committee). The funding agencies' review of these guideline papers, following JGC approval but prior to submission for publication, was limited to whether to endorse or reject the body of work. See Fig. 1 for an outline of key steps in the process of developing the systematic review and evidence-based guidelines.

\section{Acknowledgments}

We acknowledge the JGC of the AANS and CNS for the members' reviews, comments, and suggestions; Pamela Shaw, research librarian, for her assistance with the literature searches; Kevin Boyer for his assistance with data analysis; and Sue Ann Kawecki for her assistance with editing. We also acknowledge the following peer reviewers for their contributions: Timothy C. Ryken, M.D., David P. Adelson, M.D.; Brian L. Hoh, M.D.; Mark D. Krieger, M.D.; Mark E. Linskey, M.D.; Jeffrey J. Olson, M.D.; Patricia Raskin, M.D.; Krystal L. Tomei, M.D.; and Monica Wehby, M.D.

\section{Disclosure}

The authors report no conflict of interest. All Task Force members declared any potential conflicts of interest prior to beginning work on this systematic review and evidence-based guidelines.

Sources of Support: The systematic review and evidence-based clinical practice guidelines were funded exclusively by the AAANS/ $\mathrm{CNS}$, which received no funding from outside commercial sources to support the development of this document unless otherwise stated in each part of these guidelines.

Author contributions to the study and manuscript preparation include the following. Conception and design: AANS/CNS Joint Section on Pediatrics. Acquisition of data: both authors. Analysis and interpretation of data: Flannery. Drafting the article: Flannery. Critically revising the article: both authors. Reviewed submitted version of manuscript: both authors. Approved the final version of the manuscript on behalf of both authors: Flannery. Administrative/technical/material support: both authors. Study supervision: Flannery.

\section{References}

1. Berry JG, Toomey SL, Zaslavsky AM, Jha AK, Nakamura $\mathrm{MM}$, Klein DJ, et al: Pediatric readmission prevalence and variability across hospitals. JAMA 309:372-380, 2013 (Erratum in JAMA 309:986, 2013)

2. Eddy DM, Hasselblad V, Shachter RD: Meta-Analysis by the 


\section{Part 1: Introduction and methodology}

Confidence Profile Method: The Statistical Synthesis of Evidence. Boston: Academic Press, 1992

3. Klimo P Jr, Thompson CJ, Baird LC, Flannery AM: Pediatric hydrocephalus: systematic literature review and evidencebased guidelines. Part 7: Antibiotic impregnated shunt systems versus conventional shunts in children: a systematic review and meta-analysis. J Neurosurg Pediatr 14 Suppl:53-59, 2014

4. Klimo P Jr, Van Poppel M, Thompson CJ, Baird LC, Duhaime AC, Flannery AM: Pediatric hydrocephalus: systematic literature review and evidence-based guidelines. Part 6: Preoperative antibiotics for shunt surgery in children with hydrocephalus: a systematic review and meta-analysis. J Neurosurg Pediatr 14 Suppl:44-52, 2014

5. Murphy MK, Black NA, Lamping DL, McKee CM, Sanderson $\mathrm{CF}$, Askham J, et al: Consensus development methods, and their use in clinical guideline development. Health Technol Assess 2:i-iv, 1-88, 1998

6. Ransohoff DF, Pignone M, Sox HC: How to decide whether a clinical practice guideline is trustworthy. JAMA 309:139_ 140,2013

7. Simon TD, Riva-Cambrin J, Srivastava R, Bratton SL, Dean JM, Kestle JR: Hospital care for children with hydrocephalus in the United States: utilization, charges, comorbidities, and deaths. J Neurosurg Pediatr 1:131-137, 2008

8. Williams MA, McAllister JP, Walker ML, Kranz DA, Bergsneider M, Del Bigio MR, et al: Priorities for hydrocephalus research: report from a National Institutes of Health-sponsored workshop. J Neurosurg 107 (5 Suppl):345-357, 2007

Manuscript submitted June 25, 2014.

Accepted July 7, 2014.

Please include this information when citing this paper: DOI: 10.3171/2014.7.PEDS14321.

Address correspondence to: Ann Marie Flannery, M.D., Department of Neurological Surgery, Saint Louis University, 3565 Vista Ave., St. Louis, MO 63110. email: flanneam@slu.edu. 